\title{
Scalar fluctuation and its dissipation in turbulent reacting flows
}

N. Swaminathan ${ }^{1, \text { a) }}$ and N. Chakraborty ${ }^{2, b)}$

${ }^{1)}$ Hopkinson Lab, Department of Engineering, Cambridge University, Cambridge, $U K$

${ }^{2)}$ School of Engineering, Newcastle University, Newcastle-Upon-Tyne, $U K$

(Dated: 27 December 2020, revised, original submission on 2/Dec/2020)

The dissipation rate of a scalar variance is related to mean heat release rate in turbulent combustion. Mixture fraction is the scalar of interest for non-premixed combustion and a reaction progress variable is relevant for premixed combustion. A great deal of work is conducted in past studies to understand the spectra of passive scalar transport in turbulent flows. A very brief summary of these studies to bring out salient characteristics of passive scalar spectrum is given first. Then, the classical analysis of reactive scalar spectrum is revisited in the lights of recent understanding gained through analyzing the scalar spectrum deduced from direct numerical simulation data of both non-premixed and premixed combustion. The analysis shows that the reactive scalar spectral density in premixed combustion has a dependence on Karlovitz and Damköhler numbers, which comes through the mean scalar dissipation rate appearing in the spectral expression. In premixed combustion, the relevant scale for the scalar dissipation rate is shown to be of the order of the chemical length scale and the dissipation rate is not influenced by the scales in the inertial-convective range unlike for the passive scalar dissipation rate. The scalar fluctuations produced near the chemical scales cascade exponentially to larger scales. These observations imply that the passive scalar models cannot be extended to premixed combustion.

\footnotetext{
a)Electronic mail: ns341@cam.ac.uk

b)Electronic mail: nilanjan.chakraborty@newcastle.ac.uk
} 


\section{INTRODUCTION}

The mixing of fuel and air in non-premixed turbulent combustion plays vital role in sustaining the combustion processes and the rate of this mixing is influenced by turbulence, molecular diffusion and also combustion itself. The influence of combustion comes through thermal expansion effects on turbulence and turbulence-chemistry interaction. Both turbulence and combustion are multiscale phenomena and hence one can expect a cross-scale influence of one another. However, Bilger ${ }^{1}$ pointed out that the mixing rate at small scales directly influences combustion by deducing that the instantaneous reaction rate per unit volume for species $i$ is given by $\dot{\omega}_{i}=-N_{\xi} d^{2} Y_{i} / d \xi^{2}$, where $\xi$ is a chemically conserved scalar, known as mixture fraction. The mass fraction of species $i$ is $Y_{i}$ and the small-scale mixing rate is denoted by $N_{\xi}$ and is defined as $N_{\xi}=\mathscr{D}(\nabla \xi \cdot \nabla \xi)$, where $\mathscr{D}$ is the molecular diffusivity which is taken to be the same for all the species involved in the combustion process. This small scale mixing rate, $N_{\xi}$, is commonly known as the scalar dissipation rate of the mixture fraction. Bilger ${ }^{2}$ showed that the combustion and associated heat release effects are local in non-premixed combustion and thus the theories and models developed for passive scalar transport in non-reacting turbulence can be employed for non-premixed combustion.

The central role of small-scale mixing in turbulent premixed combustion was shown by Bray. 3,4 The rate of this mixing between hot and cold mixtures is signified by the scalar dissipation rate of reaction progress variable, $c$, which may be defined using either a scalar mass fraction or temperature. Also, the reaction progress variable is not a conserved or passive scalar like $\xi$ and its dissipation rate is defined as $N_{c}=\mathscr{D}_{c}(\nabla c \cdot \nabla c)$. Hereafter, we shall drop the subscript for $\mathscr{D}$ and interpret it in the context of the scalar under consideration. The scalar dissipation rate is not only influenced by turbulence but also by combustion, molecular diffusion and the interactions among these physical processes. ${ }^{5-7}$ Hence, the theory and modeling of passive scalar transport in turbulence cannot be extended directly to premixed combustion and this shall be further asserted through the scalar spectrum analysis discussed in the next section. Also, the heat release in premixed combustion affects the kinematic relationship between the turbulence and scalar fields. ${ }^{8-11}$ The scalar gradient vector aligns with the most extensive principal strain rate in premixed combustion when the heat release effects overwhelms turbulent straining whereas it aligns with the most compressive prin-

cipal strain rate in non-premixed combustion ${ }^{12}$ and for passive scalar transport. ${ }^{13-15}$ This possible change in the alignment brings leading order effects to the scalar mixing (scalar dissipation rate) physics and its modeling. ${ }^{16,17}$ 
Typically, the scalar dissipation rate of a passive scalar such as mixture fraction is estimated using a linear relaxation model written as $\widetilde{N}_{\xi} \simeq C_{\xi} \sigma_{\xi}^{2} / \tau_{t}$, where the $\sim$ represents density weighted average for Reynolds-Averaged-Navier-Stokes (RANS) calculations or density weighted filtered value for a large eddy simulation (LES). The symbols $\sigma_{\xi}^{2}$ and $\tau_{t}$ represent variance of $\xi$ and turbulence timescale respectively. This model introduced first by Corrsin ${ }^{18}$ is valid when the molecular diffusion is smaller than the turbulent dispersion of scalar concentration. ${ }^{19}$ This condition is satisfied in many reacting and non-reacting turbulent flows and thus, the above linear relaxation model is commonly used for RANS calculations. Also, it can be extended to subgrid dissipation rate in LES with dynamic procedure ${ }^{20}$ to estimate the model constant, $C_{\xi}$, with an understanding that $\sigma_{\xi}^{2}$ and $\tau_{t}$ implying the subgrid variance and turbulence timescale respectively. The inadequacy of this model for premixed combustion was demonstrated in past studies for RANS ${ }^{7,21}$ and LES$^{22-25}$ calculations. This is because of the strong influence of combustion effects on turbulence, scalarturbulence interaction and their feedback on combustion.

A reasonable way to include these close coupling into the modelling of the scalar dissipation rate is to deduce a model through careful analysis of the transport equation for $N_{c}$. Furthermore, the scalar dissipation rate is required in almost all of combustion modeling approaches in one form or another. For example, the mean or filtered scalar dissipation rate is required for eddy breakup model and the approaches like conditional moment closure ${ }^{26}$ and transported joint scalar PDF $27-31$ (probability density function) require conditional dissipation rate. The flame surface density is also known to be related to the conditional dissipation rate. ${ }^{32}$ Professor $\mathrm{O}^{\prime}$ Brien has made seminal contributions to the topic of scalar mixing in turbulent flows with and without chemical reactions, specifically in the views of PDF approach. Hence, we think that it is appropriate to revisit the problem of scalar mixing in turbulent reacting flows to bring together recent understandings and developments.

The aim, here, is not to do an exhaustive and broader review but to revisit the classical ideas put forward by $\mathrm{O}^{\prime}$ Brien, Corrsin and others for passive and reactive scalar spectra, and thereby the scalar dissipation rate, in combusting flows of engineering interest. This is conducted specifically in the light of recent findings on the scalar mixing physics in reactive flows. This paper is organised as follows. The scalar spectra are discussed in the next section. The scalar dissipation rate appearing in the scalar spectrum needs to be modeled which is discussed in Section III. Some new interpretation of reactive scalar spectrum is discussed in Section IV and the conclusions are summarised in the final section. 


\section{SCALAR AND ITS DISSIPATION SPECTRA}

The spectral density, $E_{q}(k, t)$, of scalar fluctuation contains many important characteristics of the scalar field, $q$, representing either the mixture fraction or reaction progress variable. The integral of this spectral density over all the possible wavenumbers, $k$, yields the scalar variance, $\sigma_{q}^{2}$ which is given by the two-point correlation function $R_{q}(\mathbf{r}=0)$. For variable density flows, this correlation function is defined as ${ }^{33}$

$$
R_{q}(\mathbf{r})=\frac{\overline{\left.\left.\left[\rho(\mathbf{x}) q^{\prime \prime}(\mathbf{x}) q^{\prime \prime}(\mathbf{x}+r)\right)+\rho(\mathbf{x}+r) q^{\prime \prime}(\mathbf{x}+r) q^{\prime \prime}(\mathbf{x})\right)\right]}}{2 \bar{\rho}}
$$

where $q^{\prime \prime}$ is the fluctuation of $q$ over its density weighted mean, $\widetilde{q}$, and $\rho$ is the local fluid density. The spectral density is given by

$$
E_{q}(k, t)=\int_{-\infty}^{\infty} \Phi_{q}(\mathbf{k}, t) \delta(|\mathbf{k}|-k) d \mathbf{k}
$$

where $\Phi_{q}$ is the spatial Fourier transform of $R_{q}$ and $\mathbf{k}$ is the wavenumber vector. The spectral density obeys the following equation when the scalar and turbulence fields are homogeneous ${ }^{18,25,34}$

$$
\frac{\partial E_{q}}{\partial t}=T(k, t)-2 \mathscr{D} k^{2} E_{q}+2 \mathscr{F}\left(\widehat{\dot{\omega}}_{q}\right)
$$

where $\mathscr{F}\left(\widehat{\hat{\omega}}_{q}\right)$ is the spectral density of the reaction related terms appearing in the transport equation for $\sigma_{q}^{2}=R_{q}(0)$ (see equation 3.40 of Kolla et al. ${ }^{33}$ ) The term $T=-\partial\left[s(k, t) E_{q}\right] / \partial k$ is the spectral transfer of scalar fluctuations from one wavenumber to another. The quantity $s E_{q}$ is the spectral flux across a wavenumber $k$.

For a passive scalar like mixture fraction, the reaction rate is zero and thus Eq. (2) becomes $\partial\left[s E_{\xi}\right] / \partial k=-2 \mathscr{D} k^{2} E_{\xi}$ for a stationary state and the right-hand-side of this expression coming from the molecular diffusive flux in $\mathbf{r}$ space is expected to contribute for large wavenumbers. Hence, this expression simplifies further for wavenumbers in the inertial-convective range, yielding $E_{\xi} \sim s^{-1}$ and $s(k)$ is obtained through physical consideration. This has been done in past studies for Schmidt numbers (defined as $S c=v / \mathscr{D}$, where $v$ is the kinematic viscosity) of the order one, larger and smaller than unity. ${ }^{35-39}$ The results of these studies are summarised in Fig. 1 and it is important to note that the above deduction is valid only for the intertial-convective and viscous-convective ranges of wavenumbers marked in this figure. ${ }^{40}$ These past studies suggested that $s \sim \varepsilon^{1 / 3} k^{5 / 3}$ and $k \sqrt{\varepsilon / v}$ for the inertial-convective and the viscous-convective ranges respectively, where $\varepsilon$ is the dissipation rate of the turbulent kinetic energy, $K$. Hence, the wellknown behaviours $k^{-5 / 3}$ and $k^{-1}$ are observed for the inertial-convective and viscous-convective 
ranges, ${ }^{35-38}$ which were also verified through direct numerical simulations (DNSs). ${ }^{40,41}$ However, for the inertial-diffusive range, where the viscous effects are negligible but the diffusive effects are important, the spectral balance is $\partial\left[s E_{\xi}\right] / \partial k=-2 \mathscr{D} k^{2} E_{\xi}$. By using the expression for $s$ in the inertial-convective range in this spectral balance, one can deduce that

$$
E_{\xi}=A_{\xi} \varepsilon^{-1 / 3} k^{-5 / 3} \exp \left[\frac{-3 A_{1}}{2}\left(\frac{k}{k_{o c}}\right)^{4 / 3}\right],
$$

where $A_{\xi}$ is a constant which is evaluated using the dissipation rate of the scalar variance, $\chi_{\xi}=2 \overline{\rho \mathscr{D}\left(\nabla \xi^{\prime \prime} \cdot \nabla \xi^{\prime \prime}\right)} / \bar{\rho}=2 \mathscr{D} \int k^{2} E_{\xi} d k$ and this gives $A_{\xi}=2 \chi_{\xi} / 3$ for the inertial-convective range. ${ }^{18,37}$ The factor $2 / 3$ is known as the Obukhov-Corrsin constant. For $k \gg k_{o c}$ (viscousdiffusive range), there is an exponential decrease of $E_{\xi}$ from $k^{-5 / 3}$ with a portion showing $k^{-17 / 3}$ behaviour. ${ }^{34}$ The wavenumber corresponding to the Obukhov-Corrsin length scale is denoted using $k_{o c}=\left(\varepsilon / \mathscr{D}^{3}\right)^{1 / 4}$ and is related to the Kolmogorov wavenumber, $k_{k}$, through $k_{o c}=k_{k} S c^{3 / 4}$. Chemically reacting turbulent flows with $S c \ll 1$ are rare for terrestrial conditions and applications. Although there are practical applications such as mixing of two liquids with chemical reactions satisfying $S c \gg 1$ condition, we limit ourselves to the case of $S c \sim O(1)$ by considering engineering and technological applications involving gaseous flows.

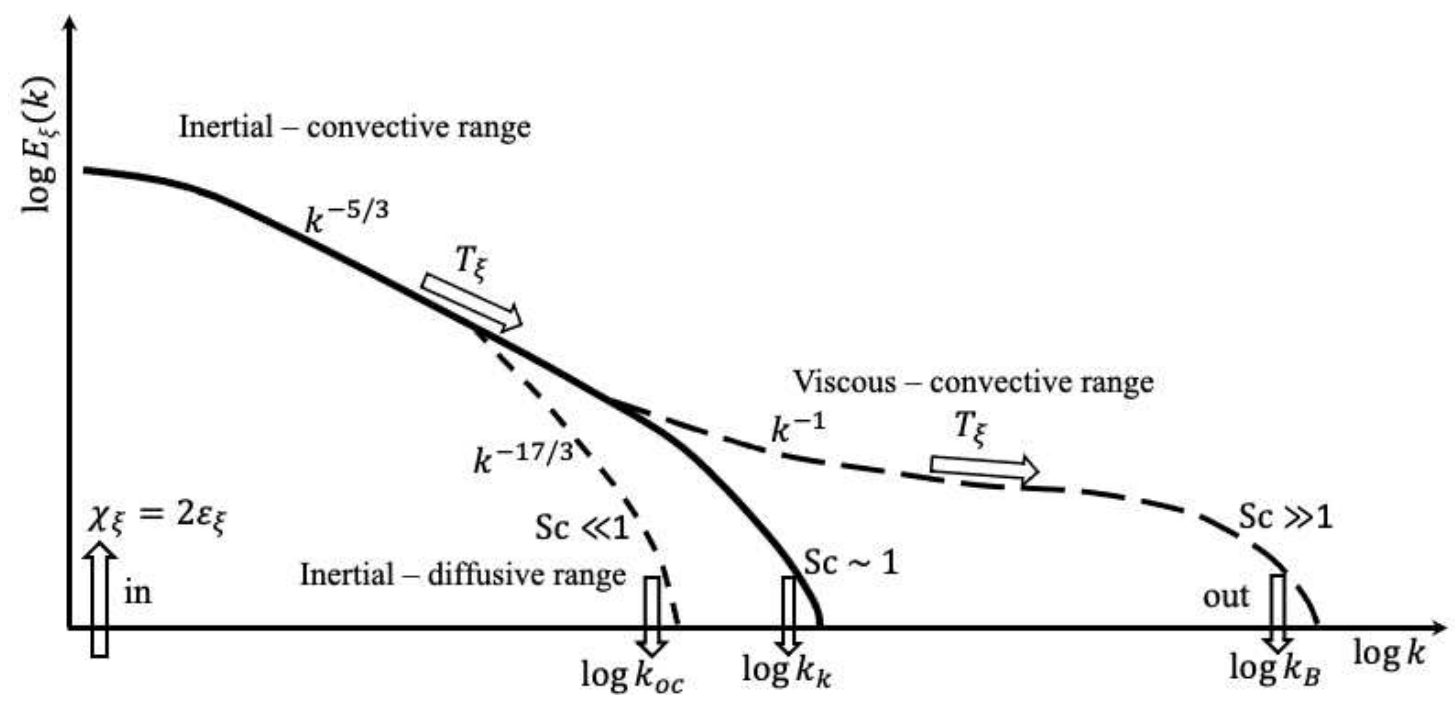

FIG. 1. Schematic of stationary passive scalar spectrum, $E_{\xi}$, for three limiting cases. ${ }^{40}$ Adopted with permission from the book Ten chapters in turbulence 2012. Copyright 2012 Cambridge University Press.

The assumption of homogeneous flow used while writing Eq. (2) can be questioned for reacting flows, specifically for premixed combustion. However, the shapes of velocity and scalar 
spectra computed using DNS data of non-premixed ${ }^{42}$ and premixed $^{33}$ flames (involving one-step and multi-step chemical kinetics) suggest that it may be reasonable to make this approximation to deduce further insights into the spectral behaviour of scalar fluctuations in reacting flows. Also, velocity and scalar dissipation spectra deduced using DNS data of premixed flames lend support for this view. ${ }^{43}$. Hence, we shall use Eq. (2) for further analysis here. However, one must be cautious in interpreting and applying the results and insights discussed in this paper and the above conditions should be borne in mind.

Since the mass fraction of a reactive scalar, $Y_{i}$, in non-premixed combustion can be expressed as a liner function of mixture fraction, if all the molecular diffusivities are the same and the chemical timescale is very much shorter than a typical turbulent timescale, one can see that $Y_{i}$ spectra are directly related to the mixture fraction spectrum. However, combustion introduces an additional length scale, which can be of the order of Kolmogorov scale or thinner. Hence, the reactive scalar spectra, despite its direct relation to the mixture fraction spectra, may not collapse together if one employs the diffusive scale such as Obukhov-Corrsin length scale which is commonly used for passive scalar spectrum. ${ }^{42}$ Perhaps, the appropriate scale to use for the diffusive-reactive range, $k \gg k_{o c}$, may be the reaction zone thickness which can be defined ${ }^{44}$ as $\delta_{r} \simeq \sqrt{2 \mathscr{D} \Delta Z / \chi_{\xi}}$, where $\Delta Z$ is the mixture fraction space thickness over which the heat release rate drops to $10 \%$ of its maximum. This kind of scaling is yet to be explored to study the scalar spectra in non-premixed combustion. However, Knaus and Pantano ${ }^{42}$ showed that the Kolmogorov scaling holds well for the inertial-convective range if one uses density weighted quantities for the scaling.

The classical works by Corrsin ${ }^{18}, \mathrm{O}^{\prime} \mathrm{Brien}^{45}$ and $\mathrm{PaO}^{34}$ showed the influence of first-order chemical reaction on the scalar spectrum under dilute conditions (heat release effects are small). Furthermore, Pao showed ${ }^{34}$ the need for the numerical solutions when introducing reversible reaction and, series and parallel reactions in the analysis but those reactions did not change the spectral characteristics. In the following analysis, we shall revisit those analyses in the light of recent understandings of reactive scalar spectra deduced using DNS data of premixed combustion with realistic finite rate chemical reactions (multi-step chemical kinetics) and large heat release rates. These DNS studies showed that the pressure-dilation work, denoted by $\overline{p^{\prime} \nabla \cdot \mathbf{u}^{\prime \prime}}$, played leading order role for the turbulent kinetic energy budget in premixed flames. ${ }^{46,47}$ Thus, the velocity spectrum depicted the due influence of this effect at the wavenumber corresponding to a laminar flame thickness along with a corresponding influence in scalar spectra also. ${ }^{33}$ However, the shape of the spectrum, specifically for wavenumbers sufficiently smaller than the wavenumber corresponding 
to the laminar flame scale (chemical length scale), remained similar to those observed in constant density flows.

For a reaction $F+v O \rightarrow(1+v) P$, where $v$ is the oxidiser mass per unit mass of fuel, with a reaction rate expression $\dot{\Omega}_{F}=-K_{r}[F][O]$, one can write $\dot{\Omega}_{F}=-[F] / \tau_{c}$ for fuel-lean combustion systems and $\tau_{c}$ is a chemical timescale for the reaction and $[F]$ is the molar density of the species $F$. Hence, approximating the combustion reaction as a first-order reaction (with respect to the species of interest) is acceptable although its overall order is two. Even if one considers the Arrhenius rate expression for $K_{r}$ then the exponential term involving temperature or $\tau_{c}$ can be estimated for the temperature, $T$, corresponding to the maximum reaction rate (or heat release rate). If one considers the full exponential dependence on temperature for $K_{r}$ then one must consider the crosscorrelation between $F$ and $T$ and the corresponding spectra under the above approximation for the reaction rate, otherwise the cross-correlations of $F-O, F-T$ and $O-T$, and their spectra must be included in the analysis. If one considers a multi-step chemical kinetics then additional crosscorrelations among scalars will arise as demonstrated by Pao. ${ }^{34}$ These additional cross-spectra and the exponential non-linear term for temperature will severely limit the analytical treatment to deduce further understanding of reactive scalar spectra. Also, it is common to express reaction rate per unit volume using a reaction progress or a regress variable in premixed combustion. Thus, the subscript $F$ used in the discussion below implies the reaction regress variable, which takes 1 in the unburnt mixture and 0 in the burnt mixture. This variable is related to the progress variable through $c=1-F$. With the above points noted, the spectral density of the reaction related term becomes $\mathscr{F}\left(\widehat{\dot{\omega}}_{F}\right)=-E_{F} / \tau_{c}$. Now, Eq. (2) gives

$$
\frac{d s E_{F}}{d k}=-2 \mathscr{D} k^{2} E_{F}-2 \frac{E_{F}}{\tau_{c}}
$$

for the stationary spectral density of $F$. It is to be noted that the dissipation contribution is also included along with the reaction related term since the coupling between reaction rate and molecular diffusion is strong in premixed combustion. We have $s \sim \varepsilon^{1 / 3} k^{5 / 3}$ for the case of our interest and thus one gets

$$
E_{F}(k)=B \varepsilon^{-1 / 3} k^{-5 / 3} \exp \left[\frac{-3 A_{2}}{2}\left(\frac{k}{k_{o c}}\right)^{4 / 3}+3 A_{2}\left(\frac{k}{k_{o c}}\right)^{-2 / 3}\left(\frac{k_{c h}}{k_{o c}}\right)^{2}\right]
$$

where $k_{c h}=\left(\mathscr{D} \tau_{c}\right)^{-1 / 2}$ is the wavenumber corresponding to the chemical length scale, which is the Zeldovich thickness. It is also worth noting that $k_{c h} / k_{o c}=K a^{-1 / 2} S c^{-3 / 4}$, where $K a=\left(k_{k} / k_{c h}\right)^{2}$ 
is the Karlovitz number. The dimensional constant $B$ can be evaluated to be $B=\chi_{F} /(3 I)$ using the identity $\chi_{F}=2 \mathscr{D} \int k^{2} E_{F} d k$, where $I$ is given by the integral

$$
I=\int_{x_{l}}^{\infty} x \exp \left[\frac{-3 A_{2}}{2} x^{2}+\frac{3 A_{2}}{x K a}\right] d x
$$

with $x=\left(k / k_{o c}\right)^{2 / 3}$. Hence, $E_{F}$ given in Eq. (5) becomes

$$
E_{F}(k)=A_{F} \chi_{F} \varepsilon^{-1 / 3} k^{-5 / 3} \exp \left[\frac{-3 A_{2}}{2 S c}\left(\frac{k}{k_{k}}\right)^{4 / 3}+\frac{3 A_{2}}{K a S c}\left(\frac{k}{k_{k}}\right)^{-2 / 3}\right],
$$

with $A_{F}=1 /(3 I)$, which is expected to be of the order one. One observes that when $K a$ becomes very large (passive scalar situation) the passive scalar spectrum, $E_{\xi}$, given earlier for $S c=1$ is recovered. It is understood that the statistical quantities appearing in Eq. (7) are density weighted averages. We shall discuss the need to have the lower limit as $x_{l}$ rather than as zero after studying the characteristics of $E_{F}$.

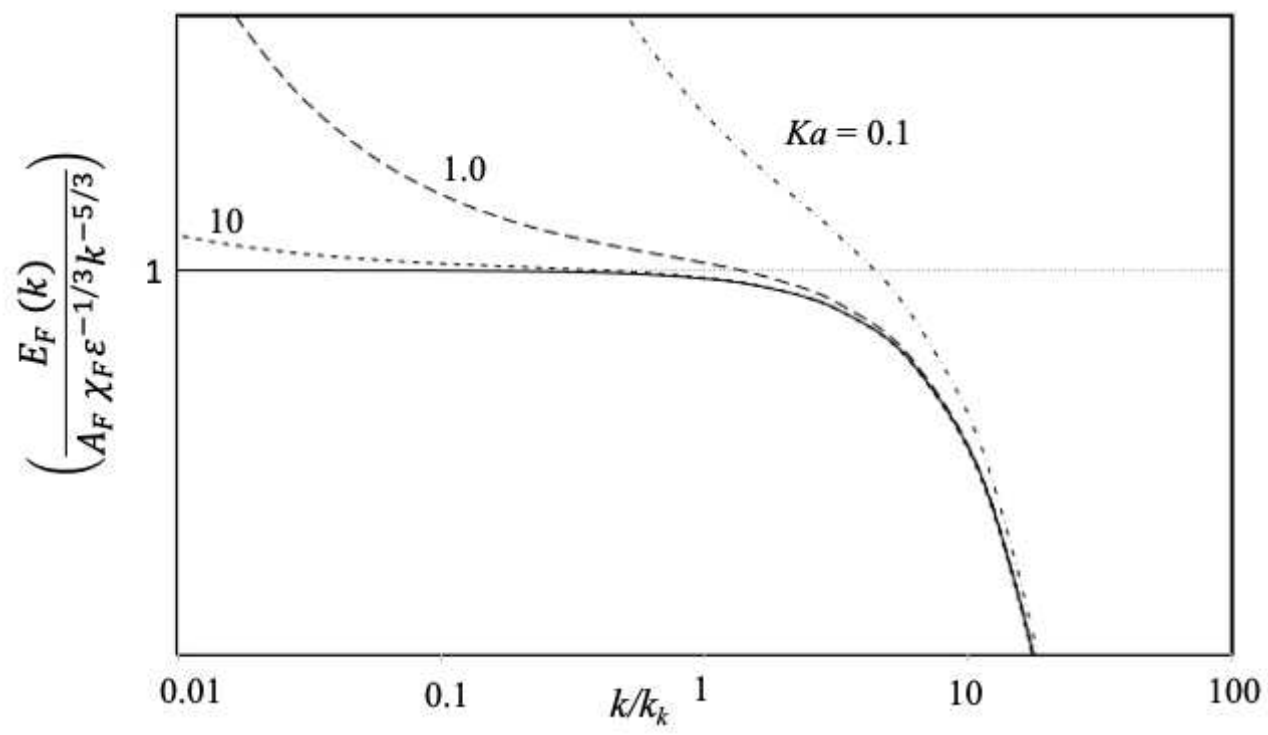

FIG. 2. Typical variation of normalised $E_{F}$ with $k / k_{k}$ in $\log$-log scale. The solid line is for non-reactive case $(K a \rightarrow \infty)$ and the other three lines are for specific $K a$ values given above and $S c=A_{2}=1$.

Figure 2 shows variation of $E_{F}(k)$ normalised using the quantities premultiplying the exponential function in Eq. (7) (also known as the compensated spectrum when $A_{F}$ is excluded from the denominator) with $k / k_{k}$, where $k_{k}$ is the Kolmogorov wavenumber. Hence, one shall see a flat line for $k / k_{k} \ll 1$ when there are no effects of molecular diffusion or combustion as shown by the solid line in Fig. 2. The diffusion effects are seen for $k / k_{k} \geq 1$ as expected for $S c=1$. Any 
deviation from this solid line is because of the exponential term arising from combustion and its effect on the scalar fluctuations at a scale 100 times larger than the Kolmogorov scale is felt quite strongly for the $K a$ values considered here when $S c$ and $A_{2}$ are unity. This influence diminishes when $k / k_{k} \geq 0.3$ for $K a=10$ and the combustion effects are felt even at a scale which is nearly $1 / 6$ th of the Kolmogorov scale for $K a=1$. This effect is extended up to $k / k_{k} \simeq 15$ for the smallest $K a$ considered here. These $K a$ values are chosen to be representative of wrinkled and corrugated flamelets, and thin reaction zones regimes of turbulent premixed combustion. ${ }^{48}$ The collapse of these three curves into a single curve depends on how the dissipation rate, $\chi_{F}$, depends on $K a$ and also the Damköhler number, $D a$, defined as the ratio of large-scale turbulence timescale to $\tau_{c}$. This turbulence timescale is given by $\tau_{t}=\int E(k) d k /\left(2 v \int k^{2} E(k) d k\right)=K / \varepsilon$, where $E$ is the turbulent kinetic energy spectrum. Furthermore, the integral $I$ may depend on $K a$ and thus one can expect $A_{F}$ to have some dependence on $K a$. Since this integral is related to the density weighted mean scalar dissipation rate, one needs to understand the leading order processes influencing the scalar dissipation rate in turbulent premixed combustion before discussing $K a$ dependency of $A_{F}$. This discussion is provided in Section IV.

It is also noted that the scalar dissipation spectrum is related to the scalar spectrum through $D_{F}(k)=2 \mathscr{D} k^{2} E_{F}(k)$ and the above comments on $E_{F}$ will carry forward to $D_{F}$ also. It has been shown in recent studies that $\chi_{F}$ has dependence on $D a$ and $K a$ and these studies are reviewed briefly in the next section to bring out the key aspects in them to further understand the reactive scalar spectrum behaviour in premixed combustion. Also, it clear from the foregoing discussion that the statistical quantities such as the scalar dissipation rate in turbulent premixed combustion cannot be modeled by simply extending the passive scalar models because the combustion effects on the scalar fluctuation are felt strongly on a wide range of scales, and is driven by chemical reactions which are small scale phenomena. Indeed, this point will become clearer in Section IV.

\section{SCALAR DISSIPATION RATE MODELING}

The scalar dissipation rate, $\chi_{F}$, appearing in Eq. (7) denotes the dissipation rate of Favre vari-

ance of $F, \sigma_{F}^{2}=\overline{\rho F^{\prime \prime} F^{\prime \prime}} / \bar{\rho}$, and it is defined as $\chi_{F}=2\left(\overline{\rho \mathscr{D} \nabla F^{\prime \prime} \cdot \nabla F^{\prime \prime}}\right) / \bar{\rho}=2 \varepsilon_{F}$. A transport equation for $\varepsilon_{F}$ was derived by Swaminathan and Bray ${ }^{7}$ and it is written as

$$
\frac{D \bar{\rho} \varepsilon_{F}}{D t}=D_{1}-D_{2}+T_{1}+T_{2}+T_{3}+T_{4}
$$


after ignoring the contributions coming from temperature dependence of $\mathscr{D}$. The operator $D / D t$ is the substantial derivative, the molecular diffusion and dissipation are denoted by $D_{1}=\nabla$. $\left(\overline{\rho \mathscr{D}} \nabla \varepsilon_{F}\right)$ and $D_{2}=2 \overline{\rho \mathscr{D}^{2}\left(\nabla \cdot \nabla F^{\prime \prime}\right)^{2}}$ respectively. The turbulent transport of the dissipation rate is represented by $T_{1}$. The effect of dilatation, when $L e=1$, is represented by $T_{2}=2 \overline{\rho \varepsilon_{F} \nabla \cdot \mathbf{u}^{\prime \prime}}$ which results from the density change across the flame front and this term generally acts as a source term for $\varepsilon_{F}$ evolution. The symbol $L e$ is the Lewis number which is defined as the ratio of thermal diffusivity to mass diffusivity. The interaction of turbulence and scalar fields denoted by $T_{3}$ has three contributions and the inner product between the fluctuating scalar gradient and turbulent strain rate, given by $T_{32}=-2 \overline{\rho \mathscr{D}\left(\nabla F^{\prime \prime} \cdot \nabla u^{\prime \prime} \cdot \nabla F^{\prime \prime}\right)}$, is the most dominant term of $T_{3}$. The influence of reaction rate is represented by $T_{4}=2\left(\overline{\mathscr{D} \nabla F^{\prime \prime} \cdot \nabla \dot{\omega}_{F}^{\prime \prime}}\right)$, where $\dot{\omega}_{F}^{\prime \prime}$ is the fluctuating mass-based reaction rate per unit volume. An order of magnitude analysis of Eq. (8) gave

$$
T_{2}+T_{32}+T_{4}-D_{2} \simeq 0
$$

as the leading order balance for high $D a$ combustion $^{7}$. This was verified through DNS data analyses for both high and low Da combustion and typical results are shown in Fig. $3 .{ }^{16}$ These results are normalised using the respective laminar flame thermal thickness, $\delta_{t h}$, unburnt mixture density $\rho_{u}$ and laminar burning velocity $S_{L}$. For fuel lean mixtures, the thermal thickness is a multiple of Zeldovich thickness, $\sqrt{\mathscr{D} \tau}$, defined earlier. The major contribution to $T_{3}$ was shown to come from the inner product term, $T_{32} \cdot{ }^{16}$ In non-reacting turbulent flows, the scalar gradient is known to align with the most compressive principal strain rate, ${ }^{13-15}$ which is also the case for mixture fraction in non-premixed combustion, ${ }^{12}$ and thus $T_{32}$ acts as a source term for $\chi_{\xi}$. On the other hand, $T_{32}$ acts as a sink for $\chi_{F}$ in high $D a$ premixed combustion because the scalar gradient aligns with the most extensive principal strain rate $8,9,11,16$ and this difference cannot be ignored.

Simple models for the four terms in Eq. (9) were also proposed and verified using DNS data in past studies. ${ }^{16,49,50}$ These models were also used to obtain an algebraic expression for $\varepsilon_{F},{ }^{17}$ which was also validated using experimental data for turbulent burning velocities. ${ }^{51}$ This model can be written as

$$
\chi_{F}=2 \varepsilon_{F} \simeq G \frac{\left(2 K_{c}^{*}-\tau C_{4}\right) D a+C_{3}}{\tau_{t}}=G \frac{\left(2 K_{c}^{*}-\tau C_{4}\right)+C_{3} / D a}{\tau_{c}}
$$

for a location with significant dissipation rate (mean reaction rate) inside the flame brush, which would be the middle of the flame brush. The symbol $G$ is a factor of the order one, $\tau$ is the heat release parameter, defined as the temperature rise, $T_{b}-T_{u}$, across the flame front divided by the unburnt mixture temperature, $T_{u}$, which has a value of 6 to 8 for typical combustion condi- 

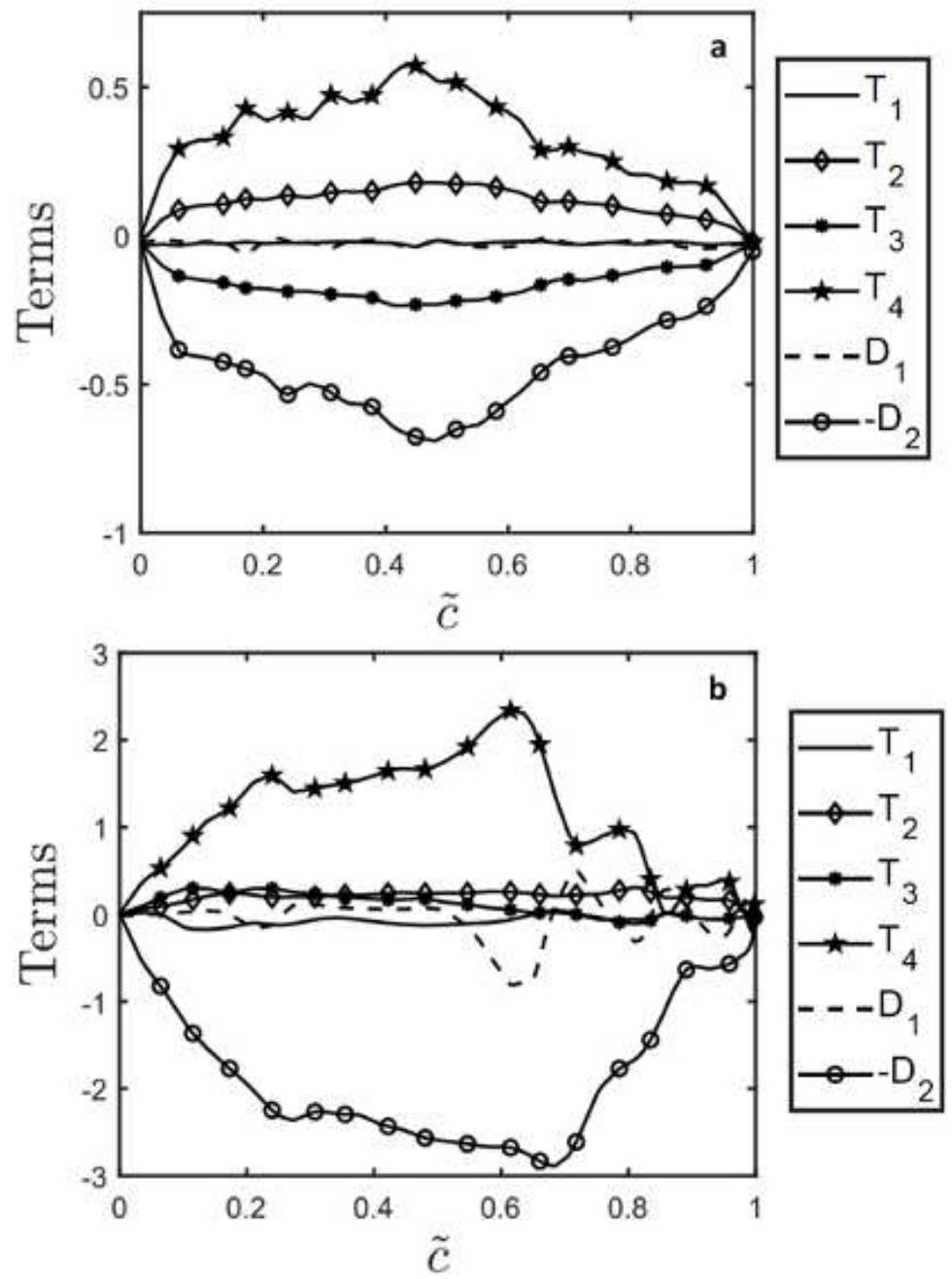

FIG. 3. Variations of $D_{1}, D_{2}, T_{1}, T_{2}, T_{3}, T_{4}$ across the flame brush, $\widetilde{C}=1-\widetilde{F}$. These terms are normalised using $\delta_{t h} /\left(\rho_{u} S_{L}^{2}\right)$ and the results are shown for two cases (a) $D a=6.8, K a=0.3$ and (b) $0.3,11 .{ }^{16} \mathrm{Re}$ produced from N. Chakraborty, J. W. Rogerson, and N. Swaminathan, "A-priori assessment of closures for scalar dissipation rate transport in turbulent premixed flames using direct numerical simulation," Phys. Fluids 20, 045106, 2008, with the permission of AIP Publishing.

tion, $K_{c}^{*} / \tau$ varying from 0.7 to 0.8 signify the contribution of $T_{2}$, and $\tau_{t}=K / \varepsilon$ is the timescale for large scale turbulence. The Damköhler number is related to $K a$ through $D a \simeq K a^{-1} \sqrt{R e_{t}}$, where $R e_{t}$ is the turbulence Reynolds number. The parameters $C_{3}=1.5 \sqrt{K a} /(1+\sqrt{K a})$ and $C_{4}=1.1(1+K a)^{-0.4}$ have dependencies on the Karlovitz number. ${ }^{17}$ These relations arise because the scalar gradient generation is driven by chemical reactions, turbulence, and turbulence-scalar interaction in premixed combustion, see Eq. (9). Indeed, the functional dependencies discussed above have come from DNS data analyses and seem to work quite well for a range of combustion 
and turbulence conditions spanning from corrugated flamelets to thin reaction zones regimes of premixed combustion. ${ }^{22-24}$ However, some minor changes to these functional dependencies were also reported in past studies when $L e$ is not unity. ${ }^{52,53}$ It is also to be noted that there are alternative approaches to model the scalar dissipation rate in premixed combustion which are reviewed by Chakraborty et al..$^{54}$

\section{DISCUSSION}

By substituting Eq. (10) into Eq. (7) one gets

$$
\begin{gathered}
\frac{E_{F} \tau_{t}}{\widehat{A}_{F} \varepsilon^{-1 / 3} k^{-5 / 3}}=f_{1}(K a) \exp \left[\frac{-3 A_{2}}{2 S c}\left(\frac{k}{k_{k}}\right)^{4 / 3}+\frac{3 A_{2}}{K a S c}\left(\frac{k}{k_{k}}\right)^{-2 / 3}\right], \\
\text { or } \quad \frac{E_{F} \tau_{c}}{\widehat{A}_{F} \varepsilon^{-1 / 3} k^{-5 / 3}}=f_{2}(K a) \exp \left[\frac{-3 A_{2}}{2 S c}\left(\frac{k}{k_{k}}\right)^{4 / 3}+\frac{3 A_{2}}{K a S c}\left(\frac{k}{k_{k}}\right)^{-2 / 3}\right],
\end{gathered}
$$

where $\widehat{A}_{F}=A_{F} G$. Equation (10) suggests that $f_{2}(K a)$ is $\left(0.5 \tau+C_{5} K a^{3 / 2} /(1+\sqrt{K a})\right)$ and $f_{1}(K a)=D a f_{2}(K a)$. Now, it is becoming clear that the full scaling of scalar spectrum in turbulent premixed combustion involves both turbulence and chemical scales as contemplated by Kolla et al. ${ }^{33}$ The above scaling on $K a$ is based on the analysis of turbulent premixed flames of hydrocarbon- and hydrogen-air mixtures in past studies which considered statistically 1D propagating flame in spatially decaying turbulence, ${ }^{8,16,17,52,55}$ oblique flames, ${ }^{56-59}$ spherically expanding flames, ${ }^{60,61}$, slot-jet flames, ${ }^{62}$ and swirling flames. ${ }^{63,64}$ These studies used both single and multi-step chemical kinetics, and also unity and non-unity Lewis numbers. Hence, the above scaling may be considered to be quite robust. However, the ranges of $D a$ and $K a$ spans a narrow region in the corrugated flamelets and thin reaction zones regimes of turbulent combustion diagram, and hence it may be prudent to consider the above scaling as tentative because of the limited ranges of $D a$ and $K a$ values.

The constant $A_{F}$ in Eq. (7) involves the integral in Eq. (6) and this integral will not converge if $x_{l}=0$ because of the $1 / x$ in the exponent. This becomes severe for low $K a$ (combustion in wrinkled flamelets regime). In his seminal analysis, $\operatorname{Corrsin}^{18}$ suggested to consider from the 'energy-bearing' wavenumber to the end of the inertial-convective range to evaluate the parameter in the scalar spectrum. Furthermore, the reaction was taken to occur in the inertial-convective range (see his Eq. 19), which is possible only if the reaction is a unimolecular reaction. Combus- 
tion reaction is generally bi-molecular and it can be approximated to be first-order with respect to the scalar of interest as discussed in Section II.

Numerical analysis of the integral in Eq. (6) suggests the following. To keep $A_{F}$ to be of the order one, $x_{l}$ must be kept as $1.26 \mathrm{Ka}^{-1 / 3}$ which comes out to be the solution of $x_{l}-$ $\exp \left[-1.5 x_{l}^{2}-3 /\left(x_{l} K a\right)\right]=0$, where $A_{2}$ and $S c$ are takend to be 1 for simplicity and without the loss of generality. This variation of $x_{l}$ shown in Fig. 4 suggests that $k_{l} / k_{k}$ varies from 4.46 for $K a=0.1$ to 0.06 for $K a=500$ for the integral in Eq. (6) to converge (have a finite value) and to have $A_{F}$ of the order one. The values of $A_{F}$ depends weakly on $K a$ as shown in Fig. 4. If one takes the lower limit to be 100 times larger than the Kolmogorov scale, see Fig. 2, then the integral in Eq. (6) become so large implying that the scalar dissipation rate, $\chi_{F}$, becomes unphysical and $A_{F}$ becomes 0 for $K a$ below 3 and this variation of $A_{F}$ with $K a$ is also shown in Fig. 4.

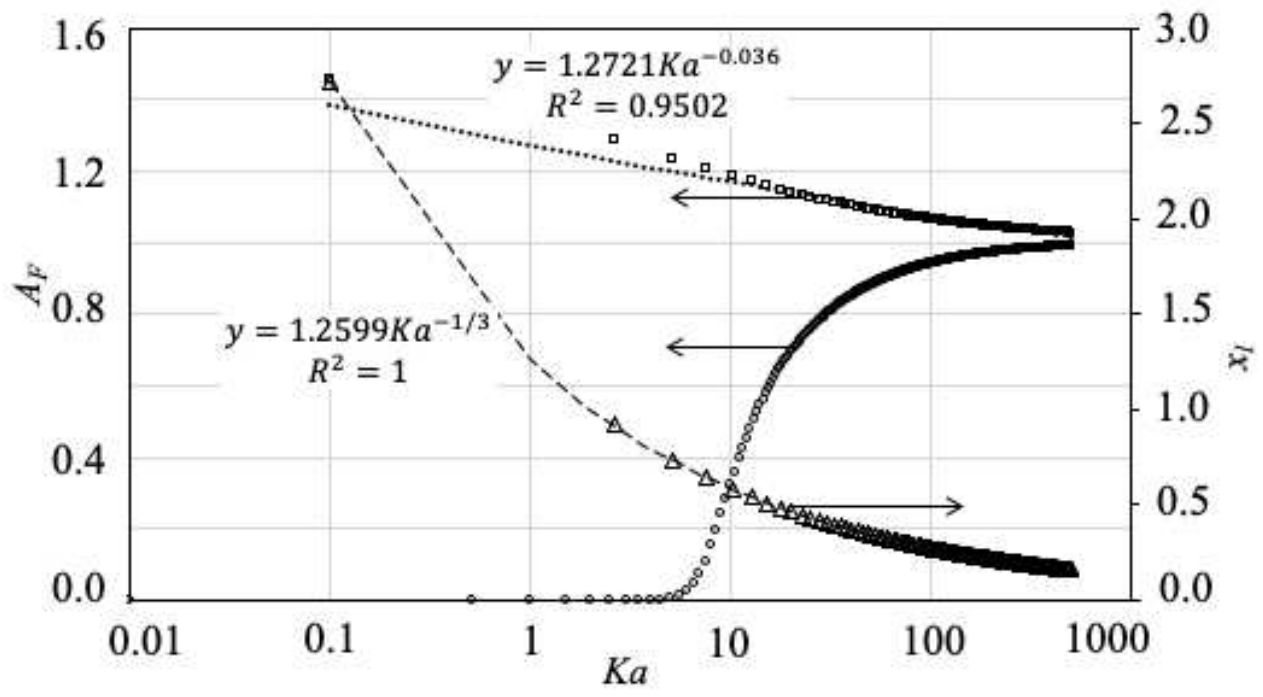

FIG. 4. Variation of $A_{F}$ and $x_{l}$ with $K a$. Symbols are from numerical computations and lines show the best fits.

The choice of having $x_{l} \sim K a^{-1 / 3}$ seem to be physically meaningful and this brings out an important point. If one translates this in terms of chemical length scale then the lower wavenumber becomes $k_{l} / k_{c h}=\sqrt{K a}\left(k_{l} / k_{k}\right)=1.41$, suggesting that the relevant largest length scale is of the order of the chemical length scale for the dissipation field. Kolla et al. ${ }^{43}$ also suggested that a chemical scale is the relevant scale for the dissipation rate by analysing the dissipation spectra deduced using DNS data of premixed flames. Furthermore, the scalar fluctuations produced by the reactions around the chemical length scale cascade exponentially to larger scales (see Eq. 11 or 12) 
as seen in Fig. 2. The rate of this cascade is larger if the combustion is stronger (small $\mathrm{Ka}$ or large $D a$ ). The length scales in the inertial-convective range and below do not influence the dissipation rate in premixed combustion. This is also signified by the leading order balance in Eq. (9) involving only the reaction rate related terms, except $T_{32}$. Furthermore, this $T_{32}$ term involves gradients of scalar and velocity fluctuations and thus it is also related to small scales. This observation holds on a physical basis also because the reactant mixture is homogeneous everywhere except in reacting regions of premixed combustion and hence turbulence alone cannot produce scalar gradients. Chemical reactions, which are small-scale phenomena, produce scalar gradients and the turbulence either enhances or hinders this production. Hence, using the passive scalar mixing based models for turbulent premixed combustion is questionable on physical grounds. This is also clear from the spectral considerations discussed in this study. This observation is highly relevant for LES of premixed combustion since SGS modeling involves small scale quantities.

\section{SUMMARY AND CONCLUSION}

The spectral characteristics of scalar fluctuations and their dissipation in turbulent combustion is of long standing interest. The seminal work by Corrsin, O'Brien, and Pao gave useful insights and raised many intriguing questions, for which there are no convincing answers yet. Spectral analysis using DNS data of non-premixed combustion showed that the Kolmogorov scal-

ing worked quite well if the density weighted quantities are used in the scaling. ${ }^{42}$ The pressure dilatation work was shown to have a due influence on the turbulent kinetic energy spectrum in premixed combustion along with a spike appearing in scalar spectrum due to reaction rate. ${ }^{33}$ However, the full scaling of the scalar spectrum couldn't be achieved and it may involve Kolmogorov scale, laminar flame thickness, Karlovitz, $K a$, and Damköhler, $D a$, numbers.

This study shows that the scalar spectrum, $E_{F}$, in premixed combustion involves Karlovitz and Damköhler numbers which are related to both chemical and turbulence scales. This is deduced using spectral analysis following the classical work of Corrsin. ${ }^{18}$ The dependence of $E_{F}$ on $K a$ and $D a$ is observed to arise through the mean scalar dissipation rate. It is also shown that the relevant scale for the dissipation rate is of the order of the chemical length scale and the dissipation rate in premixed combustion is not influenced by the scales in the inertial-convective range, which is contrastingly different from the case of passive scalar mixing in turbulence. Also, scalar fluctuations produced near the chemical scales cascade exponentially to larger scales. Although a 
tentative functional dependence on $K a$ and $D a$ is obtained in this study, which may hold in general, a suitable form of this scaling to collapse the entire scalar spectral density over a broad range of $D a$ and $K a$ values is still to be found and further analysis would be helpful in that regard.

\section{ACKNOWLEDGMENTS}

The help of Manisha Swaminathan with numerical integration of Eq. (6) and preparing Fig. 4 is acknowledged. Authors acknowledge the support of EPSRC, UK through grants EP/S025650/1, EP/R029369/1.

\section{DATA AVAILABILITY}

Data sharing is not applicable to this article as it involves theoretical analysis and no new data was created or analyzed in this study.

\section{REFERENCES}

${ }^{1}$ R. W. Bilger, “The structure of diffusion flames,” Combust. Sci. Technol. 13, 155-170 (1976).

${ }^{2}$ R. W. Bilger, "Some aspects of scalar dissipation,” Flow, Turbulence Combust. 72, 93-114 (2004).

${ }^{3}$ K. N. C. Bray, “The interaction between turbulence and combustion,” Proc. Combust. Inst. 17, 223-233 (1979).

${ }^{4}$ K. N. C. Bray, "Turbulent flows with premixed reactants," in Turbulent Reacting Flows, edited by P. A. Libby and F. A. Williams (Springer-Verlag, 1980) p. 115.

${ }^{5}$ L. Vervisch, E. Bidaux, K. N. C. Bray, and W. Kollmann, "Surface density function in premixed turbulent combustion modeling, similarities between probability density function and flame surface approaches," Phys.Fluids 7, 2496-2503 (1995).

${ }^{6}$ D. Veynante and L. Vervisch, “Turbulent combustion modelling,” Prog. Energy Combust. Sci. 28, 193-266 (2002).

${ }^{7}$ N. Swaminathan and K. N. C.Bray, "Effect of dilatation on scalar dissipation in turbulent premixed flames," Combust. Flame 143, 549-565 (2005).

${ }^{8}$ N. Swaminathan and R. W. Grout, "Interaction of turbulence and scalar fields in premixed flames," Phys. Fluids 18, 045102 (2006). 
${ }^{9}$ S. H. Kim and H. Pitsch, "Scalar gradient and small-scale structure in turbulent premixed combustion," Phys. Fluids 19, 115104 (2009).

${ }^{10}$ N. Chakraborty and N. Swaminathan, "Influence of Damköhler number on turbulence-scalar interaction in premixed flames, part I: Physical insight," Phys. Fluids 19, 045103 (2007).

${ }^{11}$ G. Hartung, J. Hult, C. F. Kaminski, J. W. Rogerson, and N. Swaminathan, "Effect of heat release on turbulence and scalar-turbulence interaction in premixed combustion," Phys. Fluids 20, 035110 (2008).

${ }^{12}$ O. Boratav, S. E. Elghobashi, and R. Zhong, "On the alignment of strain, vorticity and scalar gradient in turbulent, buoyant, nonpremixed flames," Phys. Fluids 10, 2260 (1998).

${ }^{13} \mathrm{G}$. Batchelor, "The effect of homogeneous turbulence on material lines and surfaces," Proc. R. Soc. London, Ser. A 231, 349 (1952).

${ }^{14} \mathrm{~K}$. Nomura and S. Elghobashi, "Mixing characteristics of an inhomogeneous scalar in isotropic and homogeneous sheared turbulence," Phys. Fluids A 4, 606 (1992).

${ }^{15}$ A. Garcia and M. Gonzalez, "Analysis of passive scalar gradient alignment in a simplified threedimensional case,” Phys. Fluids 18, 058101 (2006).

${ }^{16}$ N. Chakraborty, J. W. Rogerson, and N. Swaminathan, "A-priori assessment of closures for scalar dissipation rate transport in turbulent premixed flames using direct numerical simulation," Phys. Fluids 20, 045106 (2008).

${ }^{17}$ H. Kolla, J. W. Rogerson, N. Chakraborty, and N. Swaminathan, "Scalar dissipation rate modeling and its validation," Combust. Sci. Technol. 181, 518-535 (2009).

${ }^{18}$ S. Corrsin, "The reactant concentration spectrum in turbulent mixing with first order reaction," J. Fluid Mech. 11, 407 (1961).

${ }^{19}$ K. R. Sreenivasan, “Turbulent mixing: A perspective,” Proc. Nat. Acad. Sci. 116, 18175 (2019).

${ }^{20}$ C. D. Pierce and P. Moin, "A dynamic model for subgrid-scale variance and dissipation rate of a conserved scalar,” Phys. Fluids 10, 3041 (1998).

${ }^{21}$ T. Mantel and R. W. Bilger, "Some conditional statistics in a turbulent premixed flame derived from direct numerical simulations," Combust. Sci. Technol. 110-111, 393-417 (1995).

${ }^{22}$ T. Ma, Y. Gao, A. M. Kempf, and N. Chakraborty, "Validation and implementation of algebraic les modelling of scalar dissipation rate for reaction rate closure in turbulent premixed combustion," Combust. Flame 161, 3134-3153 (2014).

${ }^{23}$ D. Butz, Y. Gao, A. M. Kempf, and N. Chakraborty, "Large eddy simulations of a turbulent premixed swirl flame using an algebraic scalar dissipation rate closure," Combust. Flame 162, 
3180-3196 (2015).

${ }^{24}$ I. Langella, N. Swaminathan, and R. W. Pitz, "Application of unstrained flamelet SGS closure for multi-regime premixed combustion," Combust. Flame 173, 161-178 (2016).

${ }^{25}$ C. Dopazo, N. Swaminathan, L. Cifuentes, and X.-S. Bai, "Premixed combustion modeling," in Advanced Turbulent Combustion Physics and Applications, edited by N. Swaminathan, X.S. Bai, N. E. L. Haugen, C. Fureby, and G. Brethouwer (Cambridge University Press, 2021) Chap. 3, p. in press.

${ }^{26}$ A. Y. Klimenko and R. W. Bilger, “Conditional moment closure for turbulent combustion,” Prog. Energy Combust. Sci. 25, 595-687 (1999).

${ }^{27}$ E. E. O'Brien, “The probability density function (pdf) approach to turbulent reacting flows," in Turbulent Reacting Flows, edited by P. A. Libby and F. A. Williams (Springer-Verlag, 1980) Chap. 5, pp. 185-218.

${ }^{28}$ S. B. Pope, "Pdf methods for turbulent reacting flows," Prog. Energy Combust. Sci. 11, 119-192 (1985).

${ }^{29}$ F. Gao and E. E. O’Brien, “A large-eddy simulation scheme for turbulent reacting flows,” Phys. Fluids A. 5, 1282-1284 (1993).

${ }^{30}$ C. Dopazo, "Recent development in pdf methods," in Turbulent Reacting Flows, edited by P. A. Libby and F. A. Williams (Academic Press, 1994) Chap. 7, pp. 375-474.

${ }^{31}$ D. C. Haworth, "Progress in probability density function methods for turbulent reacting flows," Prog. Energy Combust. Sci. 36, 168-259 (2010).

${ }^{32}$ S. B. Pope, “Computations of turbulent combustion: Progress and challenges," Proc. Combust. Inst. 23, 591-612 (1991).

${ }^{33}$ H. Kolla, E. R. Hawkes, A. R. Kerstein, N. Swaminathan, and J. H. Chen, "On velocity and reactive scalar spectra in turbulent premixed flames," J. Fluid Mech. 754, 456 (2014).

${ }^{34}$ Y. Pao, "Statistical behavior of a turbulent multicomponent mixture with first-order reactions," AIAA J. 2, 1550 (1964).

${ }^{35}$ A. N. Kolmogorov, "The local structure of turbulence in incompressible viscous fluid for very large reynolds numbers,” Dokl. Akad. Nauk SSSR 30, 9 (1941).

${ }^{36} \mathrm{~S}$. Corrsin, "On the sprectrum of isotropic temperature fluctuations in isotropic turbulence," J. App. Phys. 22, 469 (1951).

${ }^{37}$ A. M. Obukhov, "Structure of temperature field in turbulence flows," Izv. Geogr. Geophys. 13, 58 (1949). 
${ }^{38}$ G. K. Batchelor, "Small-scale variation of convected quantities like temperature in turbulent fluid, part 1, General discussion and the case of small conductivity," J. Fluid Mech. 5, 113 (1959).

${ }^{39}$ Y.-H. Pao, Diffusive quantities in turbulent fluid, Ph.D. thesis, The Johns Hopkins University, Baltimore, MD (1962).

${ }^{40}$ T. Gotoh and P. K. Yeung, "Passive scalar transport in turbulence: A computational perspective," in Ten chapters in turbulence, edited by P. A. Davidson, Y. Kaneda, and K. R. Sreenivasan (Cambridge University Press, 2012) Chap. 3, pp. 87-131.

${ }^{41}$ S. Yigit, J. Hasslberger, N. Chakraborty, and M. Klein, "Effects of Rayleigh-bénard convection on spectra of viscoplastic fluids," Int. J. Heat Mass Trans. 147, 118947-1-13 (2020).

${ }^{42}$ R. Knaus and C. Pantano, "On the effect of heat release in turbulence spectra of non-premixed reacting shear layers,” J. Fluid Mech. 626, 67 (2009).

${ }^{43}$ H. Kolla, X.-Y. Zhao, J. H. Chen, and N. Swaminathan, "Velocity and reactive scalar dissipation spectra in turbulent premixed flames," Combust. Sci. Technol. 188, 1424 (2016).

${ }^{44}$ R. W. Bilger, “The structure of turbulent nonpremixed flames,” Proc. Combust. Inst. 22, 475-488 (1988).

${ }^{45}$ E. E. O'Brien, On the statistical behavior of a dilute reactant in isotropic turbulence, Ph.D. thesis, The Johns Hopkins University, Baltimore, MD (1960).

${ }^{46}$ N. Chakraborty, M. Katragadda, and R. S. Cant, "Statistics and modelling of turbulent kinetic energy transport in different regimes of premixed combustion," Flow Turb. Combust. 87, 205235 (2011).

${ }^{47}$ P. Paes and Y. Xuan, "Numerical investigation of turbulent kinetic energy dynamics in chemically-reacting homogeneous turbulence," Flow, Turbulence Combust. 101, 775 (2018).

${ }^{48}$ N. Peters, Turbulent Combustion (Cambridge University Press, 2000).

${ }^{49}$ N. Chakraborty and N. Swaminathan, "Effects of lewis number on scalar variance transport in premixed flames," Flow Turb. Combust. 87, 261 (2011).

${ }^{50}$ N. Chakraborty and N. Swaminathan, "Reynolds number effects on scalar dissipation rate transport and its modeling in turbulent premixed combustion," Combust. Sci. Technol. 185, 676 (2013).

${ }^{51}$ H. Kolla, J. W. Rogerson, and N. Swaminathan, "Validation of a turbulent flame speed model across combustion regimes," Combust. Sci. Technol. 182, 284-308 (2011). 
${ }^{52}$ N. Chakraborty and N. Swaminathan, "Effects of lewis number on scalar dissipation transport and its modelling implications for turbulent premixed combustion,” Combust. Sci. Technol. 182, 1201 (2010).

${ }^{53}$ Y. Gao and N. Chakraborty, "Modelling of lewis number dependence of scalar dissipation rate transport for large eddy simulations of turbulent premixed combustion," Num. Heat Trans. A 69, 1201 (2016).

${ }^{54}$ N. Chakraborty, M. Champion, A. Mura, and N. Swaminathan, "Scalar dissipation rate approach," in Turbulent premixed flames, edited by N. Swaminathan and K. N. C. Bray (Cambridge University Press, 2011) Chap. 2.3, pp. 74-102.

${ }^{55}$ Y. Gao and N. Chakraborty, "Scalar dissipation rate transport and its modeling for large eddy simulations of turbulent premixed combustion," Combust. Sci. Technol. 187, 362 (2015).

${ }^{56}$ Y. Minamoto, N. Fukushima, M. Tanahashi, T. Miyauchi, T. D. Dunstan, and N. Swaminathan, "Effect of flow-geometry on turbulence-scalar interaction in premixed flames," Phys. Fluids 23, 125107 (2011).

${ }^{57}$ T. Dunstan, Y. Minamoto, N. Chakraborty, and N. Swaminathan, "Scalar dissipation rate modelling for large eddy simulation of turbulent premixed flames," Proc. Combust. Inst. 34, 1193 (2013).

${ }^{58}$ Y. Gao, N. Chakraborty, T. D. Dunstan, and N. Swaminathan, "Assessment of reynolds averaged navier-stokes modeling of scalar dissipation rate transport in turbulent oblique premixed flames," Combust. Sci. Technol. 187, 1584 (2015).

${ }^{59}$ Y. Gao, Y. Minamoto, M. Tanahashi, and N. Chakraborty, "A priori assessment of scalar dissipation rate closure for large eddy simulations of turbulent premixed combustion using a detailed chemistry direct numerical simulation database,” Combust. Sci. Technol. 188, 1398 (2016).

${ }^{60}$ N. Chakraborty, M. Klein, and N. Swaminathan, "Effects of Lewis number on the reactive scalar gradient alignment with local strain rate in turbulent premixed flames," Proc. Combust. Inst. 32, 1409 (2009).

${ }^{61}$ N. Chakraborty, J. W. Rogerson, and N. Swaminathan, "The scalar gradient alignment statistics of flame kernels and its modelling implications for turbulent premixed combustion," Flow Turbulence Combust. 85, 25-55 (2010).

${ }^{62}$ N. Chakraborty, H. Kolla, R. Sankaran, E. Hawkes, J. Chen, and N. Swaminathan, "Determination of three-dimensional quantities related to scalar dissipation rate and its transport from two-dimensional measurements: Direct numerical simulation based validation,” Proc. Combust. 
Inst. 34, 1151 (2013).

${ }^{63}$ Y. Minamoto, K. Aoki, M. Tanahashi, and N. Swaminathan, "DNS of swirling hydrogeneair premixed flames," Int. J. Hydrogen Energy 40, 13604 (2015).

${ }^{64}$ G. Ghiasi, N. Doan, N. Swaminathan, B. Yenerdag, Y. Minamoto, and M. Tanahashi, “Assessment of sgs closure for isochoric combustion of hydrogen-air mixture,” Int. J. Hydrogen Energy 43, 8105 (2018). 


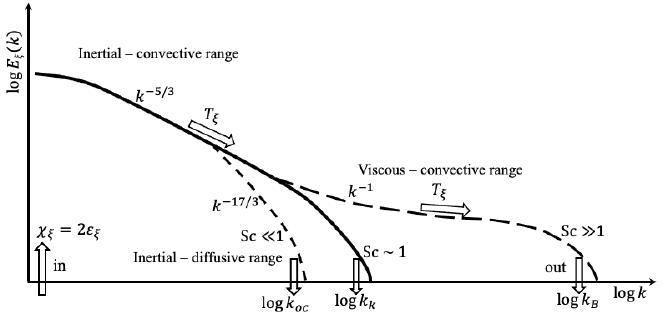




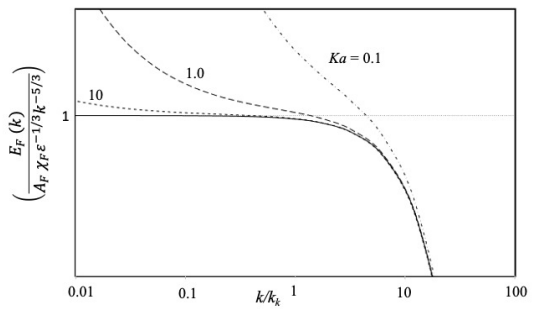



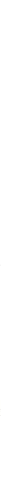


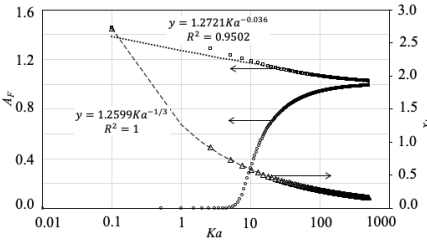

